\title{
Estrategias de promoción de alimentación saludable y de actividad física en el lugar de trabajo
}

\author{
The impact of worksite interventions promoting healthier food and/or physical activity habits \\ among employees working 'around the clock' hours: a systematic review. \\ Lassen A., Fagt S., Lennernäs M., Nyberg M., Haapalar I., Thorsen A., Møbjerg A., Beck A. \\ Food Eु Nutrition Research. 2018; 620. \\ DOI: $10.29219 /$ fnr.v62.1115 \\ DOI: $10.12961 /$ aprl.2019.22.01.6
}

\section{RESUMEN}

Se realizó una revisión sistemática de estudios aleatorios sobre el impacto de las intervenciones en el lugar de trabajo para promover una alimentación más saludable y/o la realización de ejercicio físico sobre aquellas personas que trabajan en horarios irregulares "todo el día”, es decir, fuera del horario laboral diurno ordinario. Se utilizó un diseño de estudio PICRE: Población-IntervenciónComparación-Resultados-Estudio. Las bases de datos consultadas fueron PubMed y CINAHL. Para identificar estudios adicionales a los ya incluidos, en octubre de 2017 se realizó una actualización de la búsqueda utilizando Google Scholar y la función de 'artículos relacionados' de PubMed. Se utilizó el riesgo de sesgo para evaluar la calidad de los estudios. Se incluyeron un total de siete estudios (publicados en 14 artículos) en la revisión sistemática: Dos intervenciones con un enfoque más amplio de estilos de vida, tres centrados en actividad física y dos en proporcionar una alimentación más saludable o diferentes opciones de comidas. Los estudios presentaron tamaños de la muestra desde 30 hasta 1000 y se enfocaron en una mezcla de ocupaciones, incluidos los grupos ocupacionales dominados por hombres y mujeres. Las intervenciones duraron de 2 a 12 meses. Únicamente una de ellas tuvo un seguimiento prolongado. En general, los estudios mostraron tamaños de efectos de pequeños a moderados para diversas medidas, incluyendo tanto medidas alimentarias como de actividad física, sugiriendo una eficacia aceptable en las intervenciones que involucran cambios de comportamiento a nivel comunitario. Nuestros hallazgos resaltan la necesidad de seguir desarrollando e implementando intervenciones de promoción de la salud bien diseñadas con medidas de resultado comparables e informes del tamaño del efecto. Se recomienda una combinación de estrategias de promoción de la salud para la práctica futura en esta población diana, incluyendo programas personalizados, mejorando el entorno de la alimentación y la actividad física y utilizando enfoques de estilo de vida más amplios, incluyendo el uso de estrategias participativas y de empoderamiento. Si bien se necesita más investigación en este campo, la base de conocimientos existente sobre los abordajes efectivos espera su traslado a la práctica.

\section{COMENTARIO}

Las enfermedades crónicas no transmisibles (ECNT) se reconocen como las responsables de la mayor proporción de muertes por año en el mundo, llegando a un $71 \%$ en $2018^{1}$. Parte de estas muertes podrían ser evitadas por cambios en componentes conductuales de la población, como son la inactividad física y la alimentación inadecuada, algunos de los principales factores de riesgo para el desarrollo de las ECNT².

Sin embargo, los cambios sociales de las últimas décadas están afectando de forma negativa a la práctica de actividad física y a los patrones dietéticos de la población. La industrialización de la alimentación y la apertura de los mercados parecen contribuir a la homogenización de la dieta y al aumento en la disponibilidad y en el consumo de alimentos ultraprocesados, con elevadas cantidades de sodio, azúcar y grasas saturadas y trans ${ }^{3,5}$. Además, los cambios en el mercado laboral hacen que cada vez más personas trabajen en horarios irregulares y que tengan que modificar sus prácticas alimentarias ${ }^{6}$, aumentando la demanda de comidas rápidas y realizadas fuera del hogar.

En España, aunque el patrón dietético mantuvo una relativa estabilidad durante la década del 2000 , en los últimos cuarenta años la dieta de la población ha cambiado de forma importante. Se ha reducido el consumo de alimentos básicos como cereales, aceites, hortalizas, verduras, patatas y legumbres ${ }^{7,8}$. Como consecuencia, y de forma similar a la tendencia global, se evidencia el incremento en la prevalencia de sobrepeso y obesidad que pasó de un 7,4\% en 1987 a un $17 \%$ en 2012. Al considerar un IMC mayor o igual a $25 \mathrm{~kg} / \mathrm{m}^{2}$, en este mismo año (2012) el sobrepeso y obesidad estaba presente en más de la mitad de la población adulta $(63,1 \% \text { de los hombres y } 44,1 \% \text { de las mujeres) })^{9}$.

Con vistas a afrontar esta situación, la Estrategia Mundial de la Organización Mundial de la Salud sobre Régimen Alimentario, Actividad Física y Salud, insta a los países a desarrollar estrategias que posibiliten mejorar el patrón alimentario de la población y la práctica de actividad física ${ }^{10}$. Diversos son los ámbitos de actuación necesarios para llegar a este objetivo, y entre ellos se reconoce el lugar de trabajo como un entorno capaz de fomentar conductas saludables entre la población adulta. En línea con la Estrategia de la OMS, el gobierno español ha puesto en marcha la Estrategia NAOS -Estrategia para la nutrición, actividad física y prevención de la obesidad $-{ }^{11}$. Sin embargo, la estrategia no ha incluido el lugar de trabajo con un ámbito de actuación.

La revisión sistemática publicada en "Food \& Nutrition Research" en agosto de 2018 incluyó un total de siete estudios 
reportados en 14 artículos. Los artículos incluidos presentan resultados de intervenciones en el lugar de trabajo que tienen el objetivo de promover una alimentación más saludable y/o estimular la práctica de ejercicio físico sobre aquellas personas que trabajan en horarios irregulares. La revisión sugiere efectos positivos en la dieta y/o en la práctica de actividad física, independientemente del enfoque de intervención. Además, pone de manifiesto la necesidad de mayores esfuerzos para la implementación y evaluación de intervenciones en el lugar de trabajo.

\section{Panmela Soares}

Grupo de Investigación en Salud Pública de la Universidad de Alicante y Grupo de Investigación de Nutrición en Servicios de Alimentación de la Universidad Federal de Santa Catarina (Brasil). panmela.soares@ua.es

\section{DISEÑO, IMPLEMENTACIÓN Y EVALUACIÓN} DE INTERVENCIONES ORGANIZACIONALES 13-15 de mayo de 2019, Copenhague (Dinamarca)

\section{Información:}

Linda Vänskä, NIVA, Arinatie 3A, FI-00370 Helsinki, Finlandia.

Tel.: +358405463277

E-mail: linda.vanska@niva.org

Web:https://niva.org/course/designing-implementingand-evaluating-organizational-interventions/

\section{BUENAS PRÁCTICAS DE COOPERACIÓN EN SALUD OCUPACIONAL (OHC) DE PROMOCIÓN DE LA CAPACIDAD LABORAL EN PEQUEÑAS EMPRESAS \\ 21-22 de mayo de 2019, Helsinki (Finlandia)}

\section{Información:}

Erika Gustafsson-Sundquist, NIVA, Arinatie 3A,

FI-00370 Helsinki, Finlandia.

Tel.: +358405795787

E-mail: erika.gustafsson@niva.org

Web: https://niva.org/course/good-occupational-healthcooperation-ohc-practices-of-work-ability-promotion-in-ses/

\section{REFERENCIAS}

1. Organización Mundial de la Salud. Enfermedades no transmisibles. Ginebra: OMS [actualizado 1 Jun 2018; citado 29 nov 2018]. Disponible en: http://www. who.int/es/news-room/fact-sheets/detail/noncommunicable-diseases.

2. Organización Mundial de la Salud. Informe sobre la situación mundial de las enfermedades no transmisibles. Geneva: OMS; 2014.

3. Popkin BM. Contemporary nutritional transition: determinants of diet and its impact on body composition. Proc Nutr Soc. 2011;70:82-91.

4. Popkin BM. Nutrition, Agriculture and the Global Food System in Low and Middle Income Countries. Food Policy. 2014;47:91-6.

5. Monteiro CA, Moubarac JC, Cannon G, Ng SW, Popkin B. Ultra-processed products are becoming dominant in the global food system. Obes Rev. 2013;14 Suppl 2:21-8.

6. Lindén AL, Lagnevik M, Sjöberg K, Svederberg E, Jönsson H, Nyberg M. Mat, hälsa och oregelbundna arbetstider. Lund: Department of Sociology, Lund University; 2005. 7. Varela-Moreiras G, Ávila J, Cuadrado C, Del Pozo S, Ruiz E, Moreiras O. Valoración de la dieta española de acuerdo al panel de consumo alimentario. Madrid: Ministerio de Medio Ambiente, y Medio Rural y Marino/Fundación Española de la Nutrición; 2008.

8. Del Pozo S, García V, Cuadrado C, Ruiz E, Valero T, Ávila J, et al. Valoración nutricional de la dieta española de acuerdo al panel de consumo alimentario. Madrid: Ministerio de Agricultura, Alimentacion y Medio Ambiente/Fundación Española de la Nutrición; 2012.

9. Ministerio de Sanidad Servicios Sociales e Igualdad. Informe anual del Sistema Nacional de Salud 2012. Edición revisada 2015. Madrid: Ministerio de Sanidad Servicios Sociales e Igualdad; 2015.

10. World Health Organization. Global Strategy on Diet, Physical Activity and Health. Geneva: WHO; 2004

11. Agencia española de consumo seguridad alimentaria y nutrición. Estrategia NAOS. Madrid: Ministerio de Sanidad, Servicios Sociales e Igualdad [citado 30 nov 2018]. Disponible en: http://www.aecosan.msssi.gob.es/AECOSAN/web/ nutricion/seccion/estrategia_naos.htm.

\section{CONFERENCIA INTERNACIONAL \\ DE VIBRACIONES MANO-BRAZO}

21-24 de mayo de 2019, Bonn (Alemania)

\section{Información:}

Anika Decker, Institut für Arbeitsschutz der DGUV

(IFA), Alte Heerstr. 11153757 Sankt Augustin, Alemania.

Tel.: +49 (0)301300138038

E-mail: vibration2019@dguv.de

Web: www.dguv.de/ifa/vibration2019

\section{CONFERENCIA INTERNACIONAL DE BIENESTAR EN EL TRABAJO}

22-24 de mayo de 2019, París (Francia)

Información:

E-mail: waw2019@inrs.fr

Web: www.inrs-waw2019.fr/home!en

\section{CONFERENCIA CIENTÍFICA: \\ LOS RIESGOS BIOLÓGICOS}

5-6 de junio de 2019, Nancy (Francia)

Información:

E-mail: risquesbiologiques2019@inrs.fr

Web: https://www.inrs-risquesbiologiques2019.fr/home!fr 